\title{
The mortality of children with Type 1 (insulin-dependent) diabetes mellitus in Norway, 1973-1988
}

\author{
G. Joner ${ }^{1}$ and S. Patrick ${ }^{2}$ \\ ${ }^{1}$ Department of Internal Medicine, Aker University Hospital, Oslo, Norway \\ ${ }^{2}$ Department of Epidemiology, Graduate School of Public Health, University of Pittsburgh, Pittsburgh, Pennsylvania, USA
}

\begin{abstract}
Summary. The mortality status of all individuals in Norway with the onset of Type 1 (insulin-dependent) diabetes mellitus from 1973 through 1982 and age at onset below 15 years was determined as of 1 July 1988 . Of the 1908 cases included in the follow-up, 20 had died (15 males and 5 females) and 10 had emigrated. A twofold increased risk for early mortality was exhibited among this cohort. Life-table analyses did not find sex or age at onset of Type 1 diabetes to be statistically significant predictors of survival when controlling for diabetes duration. A review of death certificates revealed that accidents and suicides accounted for $40 \%$ of the deaths in the total cohort and that this cause of death occured only among male subjects. Acute diabetes related complications were the underlying causes of death for $35 \%$ of the
\end{abstract}

subjects. Diabetic renal disease and death by cardiovascular disease were not documented in this young cohort with a maximum age of 30 years and maximum diabetes duration of 15.5 years. This is the first mortality report of a population-based registered cohort of Type 1 diabetic patients for Norway. While still being at increased risk for premature death, this cohort appears to be at decreased risk of early death when compared to a cohort of young diabetic patients from Oslo, Norway diagnosed in 19251955 , suggesting improvements in the survival of individuals with Type 1 diabetes in Norway.

Key words: Type 1 (insulin-dependent) diabetes mellitus, mortality, causes of death, accidents and suicide, Norway, population-based registry.
In spite of dramatic increases in the life expectancy of children diagnosed with Type 1 (insulin-dependent) diabetes mellitus since the introduction of insulin therapy [1], studies continue to confirm an increased mortality among subjects with Type 1 diabetes compared to healthy subjects. This increased risk for death at a given age appears to be of a seven-fold magnitude in the United States [2].

Recently, major cross-country differences in the mortality associated with Type 1 diabetes have been identified in a comparative study of cohorts of individuals with Type 1 diabetes in Finland, Israel, Japan, and the United States (as represented by Allegheny County, Pennsylvania) [3]. The reasons for these differences, however, are not known. The present study is the first to investigate the mortality of young diabetic patients in Norway and is based upon a nationwide population-based registry covering the time period from 1973 through 1982.

\section{Subjects and methods}

All new-onset cases of Type 1 diabetes occurring in Norwegian children age $0-14$ years during the time period 1 January 1973 to 31 December 1982 were registered retrospectively [4]. As described earlier, the data were collected by contacting all the paediatric and medical hospital departments in Norway for information on all new patients aged $0-14$ years with Type 1 diabetes during the study time period. The National Insurance Institution was used as a secondary source for the ascertainment of cases. The retrospective registry identified 1914 new individuals with Type 1 diabetes and achieved a case ascertainment level greater than $98 \%$ [4].

Of this cohort of 1914 Type 1 diabetes subjects, four were omitted from subsequent mortality analyses because of uncertainties regarding the exact date of diagnosis. Two subjects were considered to have diabetes secondary to other chronic conditions and, thus, were excluded, leaving a cohort of 1908 individuals. Ten diabetic subjects emigrated from Norway, and mortality follow-up within other countries was not possible. The mortality status of the 1908 subjects was determined by matching the unique personal identification number assigned to each resident of Norway to the vital statistics data collected throughout the country. Follow-up approached $100 \%$.

\section{Statistical analysis}

Life-table analyses were employed to examine the survival of the Norwegian cohort by duration of diabetes, sex, age at onset, and year of diagnosis. A Cox proportional hazards regression model was used to analyse the effects of covariates (age at diagnosis, sex, and diabetes duration) on the survival of the cohort [5]. Standardized mortality ratios (SMR) for all-cause mortality were calculated com- 
Table 1. Demographic characteristics of the 1914 Type 1 (insulindependent) diabetic subjects identified in children $0-14$ years old, 1973-1982, in Norway

\begin{tabular}{lrr}
\hline Characteristic & $n$ & $\%$ \\
\hline Sex & 1040 & 54.3 \\
$\quad$ Male & 874 & 45.7 \\
Female & & \\
Year of Birth & 179 & 9.4 \\
1958-1962 & 617 & 32.2 \\
$1963-1967$ & 733 & 38.2 \\
$1968-1972$ & 321 & 16.8 \\
1973-1977 & 64 & 3.3 \\
1978-1981 & & \\
Year of diabetes onset & 881 & 46.0 \\
1973-1977 & 1033 & 54.0 \\
1978-1982 & & \\
Age at onset (years) & 343 & 17.9 \\
$\quad<5$ & 696 & 36.4 \\
5- 9 & 875 & 45.7 \\
10-14 & & \\
Status & 1884 & 98.5 \\
Alive & 20 & 1.0 \\
Dead & 10 & 0.5 \\
Emigrated & & \\
\hline
\end{tabular}

Table 2. Age-specific mortality rates per 100,000 person-years of diabetes duration ( $95 \%$ confidence intervals by Poisson distribution)

\begin{tabular}{ll}
\hline Age attained $^{a}$ & Age-specific mortality rate \\
\hline$<5$ years & 150 \\
& $(4-833)$ \\
$5-9$ & 31 \\
& $(0.8-172)$ \\
$10-14$ & 79 \\
& $(26-184)$ \\
$15-19$ & 170 \\
& $(82-313)$ \\
$20-24$ & 91 \\
& $(11-330)$ \\
$25-29$ & 364 \\
& $(9-2027)$ \\
Total & 108 \\
& $(66-166)$ \\
\hline
\end{tabular}

a Due to cases entering and exiting the study at different times, this age group refers to the maximum age reached by a subject, either by living until the end of the study or by emigrating from Norway or dying

paring the mortality rates of the diabetic cohort to that of the general population in Norway from 1970-1986 [6] using the Occupational Cohort Mortality Analysis Program (OCMAP) by Marsh and Preininger [7].

The cause-specific death information was gathered by obtaining the International Classification of Diseases (ICD) code for the underlying cause of death listed on the death certificate registered with the Central Bureau of Statistics in Norway. During the time period of this study the ICD codes in use were either Revision 8 or 9. For clarity, all codes were translated into the ICD 9 codes [8,9]. Mortality status was determined as of 1 July 1988.

Due to the limited number of deaths, the causes of death were grouped into three groups: acute diabetes complications, accidents and suicides, and other.

\section{Results}

\section{All cause mortality}

The longest duration of Type 1 diabetes that could have been achieved at the time of follow-up was 15.5 years and the oldest possible attained age was 30 years (a 14.5-yearold diagnosed in the beginning of 1973 and followed until July 1988), thus the cohort was very young. Table 1 presents the demographic characteristics of the 1914 Norwegian cases. During the follow-up period 20 individuals died ( 15 males and 5 females), yielding a crude mortality rate of $108 / 100,000$ person-years. Death at diabetes onset occurred in only one case. Table 2 presents the age-specific and age-adjusted mortality rates per 100,000 personyears of diabetes. The age-specific SMRs by sex for the Norwegian cohort are given in Table 3. The overall SMR for all-cause mortality was 207 (95\% confidence interval 126-319).

\section{The effects of attained age and sex}

Comparing the Type 1 diabetic cohort to the general population in Norway, cases exhibited a significant twofold increase in all-cause mortality. The age group at highest risk for early mortality was the 10-14-year-old age group (SMR =339), with the 15-19-year-old age group also demonstrating a significantly increased SMR of 251 . When comparing SMRs by sex and age, male subjects exhibited significantly increased mortality in the 10-14-yearold age group (SMR $=392)$ and overall $(\mathrm{SMR}=215)$ and females demonstrated a statistically increased SMR only for the 15-19-year-old age group (SMR =434). As mentioned above, multivariate analysis did not find sex to be a significant predictor of mortality.

\section{The effects of diabetes duration}

After ten years duration, $0.46 \%$ of the cohort had died. By 15 years duration, roughly $1 \%$ of the cohort was deceased. There were no differences in the life-table results by sex $(p>0.19)$. Similarly, no statistically significant differences in mortality were seen in relationship to age at diagnosis $(p>0.1)$. Using the Cox proportional hazards regression model with mortality as the dependent variable and age at diagnosis, sex, and diabetes duration as covariates, duration was highly significant $(p<0.0001)$. The length of diabetes duration, however, was found to be negatively associated with mortality (i.e. the mean duration of the cases that died was 5.45 years, compared to a mean duration of 10.28 years for those cases who survived until 1 July 1988). This relationship held even when cases whose duration was less than one year were omitted.

\section{Cause-specific mortality}

Table 4 presents the underlying causes of death exhibited by this cohort by cause-group and ICD code. 


\section{Acute diabetes related complications}

Death due to acute diabetes related complications accounted for seven of twenty deaths, representing $35 \%$ of the total mortality, $27 \%$ of the male and $60 \%$ of the female deaths. Deaths in this group included ICD 9th Revision codes 250.2 and 250.8 .

\section{Accidents and suicides}

This cause-specific group covered the majority (40\%) of the deaths ( $53 \%$ of deaths in males). No females were recorded as dying of accident or suicide. There were three deaths due to traffic accident. In only one event the diabetic subject was the driver. There were three accidents of different causes and two suicides.

\section{Other}

Five subjects died of other causes $(25 \%)$, varying from asthma to a congenital renal malformation with uraemia. Two of these deaths were sudden and unexpected (mors subita).

\section{Discussion}

This paper is the first to report the mortality of a young population-based cohort of individuals with Type 1 diabetes in Norway. The causes of death were determined by ICD code listed on the death certificate, even if this is known to be a somewhat crude classification of the causes of death. This study has confirmed reports from other countries $[3,10,12]$ that individuals with Type 1 diabetes are at an increased risk of dying at a given age compared to the general population. This increased risk in Norway, however, appears to be small.

Because of differences in methodology, mainly regarding the criteria for the establishment of the diabetic cohort and the manner of follow-up, few mortality studies on Type 1 diabetes are directly comparable. By evaluating groups of similar initial registry criteria and duration of diabetes, however, some comparisons can be made.

Dorman et al. [2] reported the mortality experience of a cohort of 1966 Type 1 diabetic patients diagnosed at the Children's Hospital of Pittsburgh (CHP) and found that after 10 years duration $3 \%$ of the cohort had died, compared to $0.46 \%$ of the cohort in Norway. The majority of deaths in this cohort occurred after 15 years duration of diabetes. Renal disease and cardiovascular deaths were an important cause of death in the CHP-study; comparatively accidents and suicide represented the majority of deaths in the Norwegian cohort. A report from Diabetes Epidemiology Research International (DERI-group) [3] on the mortality of individuals with Type 1 diabetes in Finland, Israel, Japan and the United States has documented wide differences in the risk for premature death.

While the oldest age possibly attained by a member of the DERI cohorts is 37 years, compared to 30 in the Nor-
Table 3. Age-specific standardized mortality ratios and $95 \%$ confidence intervals (Poisson distribution)

\begin{tabular}{lclc}
\hline Age attained $^{2}$ & Males & Females & Total \\
\hline$<5$ years & 120 & - & 79 \\
& $(3-669)$ & & $(2-440)$ \\
$5-9$ & 181 & - & 99 \\
& $(4-1008)$ & & $(2-551)$ \\
$10-14$ & 392 & 219 & 339 \\
& $(107-1003)$ & $(5-1220)$ & $(110-790)$ \\
$15-19$ & 196 & 434 & 251 \\
& $(72-427)$ & $(118-1111)$ & $(120-462)$ \\
$20-24$ & 149 & - & 117 \\
& $(18-538)$ & & $(14-422)$ \\
$25-29$ & 638 & - & 469 \\
& $(16-3554)$ & & $(12-2612)$ \\
Total & 215 & 186 & 207 \\
& $(120-355)$ & $(60-434)$ & $(126-319)$ \\
\hline
\end{tabular}

a Due to cases entering and exiting the study at different times, this age group refers to the maximum age reached by a subject, either by living until the end of the study or by emigrating from Norway or dying

Table 4. The underlying causes of death as notified on the death certificate for each death occurring in the Norwegian cohort (ICD-9 codes)

\begin{tabular}{llll}
\hline Cause of death & ICD-9 Code & Sex & Age \\
\hline Acute diabetes complications & & & \\
Coma diabeticum & 250.2 & M & 5 \\
& 250.2 & F & 11 \\
& 250.2 & M & 13 \\
& 250.2 & M & 16 \\
Diabetes with insulin shock & 250.8 & M & 12 \\
& 250.8 & F & 19 \\
& 250.8 & F & 19 \\
Accidents and suicide & & & \\
Traffic accident & E819.9 & M & 17 \\
& E816.0 & M & 17 \\
Avalanche & E814.7 & M & 21 \\
Hypothermia & E909 & M & 11 \\
Explosion & E901 & M & 11 \\
Suicide & E923.1 & M & 26 \\
Suicide & E950.0 & M & 18 \\
Other & E952 & M & 23 \\
Asthma & & & \\
Acute pyelonephritis & 493 & M & 13 \\
Mors subita & 590.1 & F & 15 \\
Congenital renal malformation & 753.3 & M & $<1$ \\
& & F & 16 \\
\hline
\end{tabular}

wegian cohort, a review of the age-specific mortality rates up to age 30 for the United States, Finland, Japan, Israel and Norway reveals that Japan has the highest total mortality rate, with the United States, Finland and Norway having similar rates and Israel having the lowest rate. In Finland [11], but not in the other countries, males had a higher death rate than females. This sex discordance was not observed in the Norwegian cohort.

Data are available from Denmark on the mortality of Type 1 diabetic patients identified by a prevalence study $(n=356)$ and followed for seven years [12]. This popula- 
tion differed from the cohort in Norway in that it included individuals with Type 1 diabetes diagnosed at much older ages. Only four deaths occurred before reaching age 30 of which three were due to cardiovascular disease. While dealing with small sample sizes and the uncertainities regarding the classification of causes of death based on death certificates, it is striking that cardiovascular disease was the main cause of death for the Danish cohort and not an underlying cause in any case in the Norwegian cohort, as the background cardiovascular mortality rates are practically the same in these countries [13].

A study on the mortality of diabetic patients diagnosed between 1925-1955 from Oslo, Norway and followed until anniversary of discharge in 1961 was published by Westlund in monograph form in 1969 [14]. Although the methods employed are not directly comparable to the present study, it is the only previous study that presents mortality data for diabetic subjects for Norway. The mortality rate for young diabetic subjects $(<30$ years old at follow-up) was approximately $1000 / 100,000$ person-years (calculated by the authors). Comparing this to the rate found in the present study (108/100,000 person-years), there appears to be a major reduction in the mortality associated with Type 1 diabetes in Norway.

There are several potential reasons for this apparent considerable decrease in early mortality among patients with Type 1 diabetes in Norway. Factors such as improved physician and patient education regarding diabetes, easy access to free, high quality health care, and technological advances in glucose monitoring, diabetes care and treatment of diabetes complications are likely to have all played a role in the lengthened survival of individuals with Type 1 diabetes in Norway.

In summary, individuals diagnosed with Type 1 diabetes mellitus prior to age 15 in Norway have a twofold risk of dying before age 30 as the background population; a risk similar to that reported from Finland and Israel but lower than the United States and Japan. Accidents and suicides comprise the majority of the deaths, and of particular importance is that the current mortality rates suggest a major reduction in early mortality among individuals with Type 1 diabetes between 1925 and 1988 in Norway. As the major causes of death still claiming lives are preventable, it is hoped that an even greater decrease in mortality will be seen over the next decade.

Acknowledgements. Mr. R. Vergona (Diabetes Research Center, University of Pittsburgh) is sincerely thanked for his assistance in computer programming. Research support came from National Institutes of Health grant \# R01 AM35905 (Diabetes Epidemiology Research International Mortality Study), The Norwegian Research Council for Science and the Humanities (NAVF) grant \#344.89/006 and a grant from the Norwegian Diabetes Association.

\section{References}

1. Marks HH (1965) Longevity and mortality of diabetics. Am J Public Health 55: 416-423

2. Dorman J, LaPorte RE, Kuller LH, Cruickshanks KJ, Orchard TJ, Wagener DK, Becker DJ, Cavender DE, Drash AL (1984) The Pittsburgh insulin-dependent diabetes mellitus (IDDM) morbidity and mortality study: Mortality results. Diabetes 33: 271-276

3. LaPorte RE and Diabetes Epidemiology Research International Study Group (1989) Mortality of Type 1 (insulin-dependent) diabetes mellitus in four countries: the diabetes epidemiology research international mortality study (abstract). Diabetologia 32: $507 \mathrm{~A}$

4. Joner G, Søvik O (1989) Increasing incidence of diabetes mellitus in Norwegian children 0-14 years of age 1973--1982. Diabetologia 32: 79-83

5. Dixon WJ, Brown MB, Engelman L, Hill MA, Jennrich RI (eds) (1986) Life tables and survivor functions and survival analysis with covariates - Cox models. In: BMDP Statistical Software Manual, Vol 2. University of California Press, Berkeley, CA, pp 689-742

6. Population Statistics (1987) Central Bureau of Statistics of Norway

7. Marsh GM, Preininger M, Ehland J, Caplan R, Bearden A, CoChien H, Paik M (1989) OCMAP and OCMAP/PC mainframe and microcomputer version 2.0 user manual. University of Pittsburgh, Pittsburgh, Pennsylvania

8. International Classification of Diseases, Eighth Revision (ICD8) (1985) Central Bureau of Statistics of Norway

9. International Classification of Diseases, Ninth Revision (ICD-9) (1986) Central Bureau of Statistics of Norway

10. Panzram G (1984) Epidemiologic data on excess mortality and life expectancy in insulin-dependent diabetes mellitus - Critical review. Exp Clin Endocrinol 83: 93-100

11. Lounamaa P, Lounamaa R, Reunanen A, Tuomilehto J (1989) Mortality among patients with juvenile onset Type 1 (insulin-dependent) diabetes (abstract). Diabetologia 32:512 A

12. Green A, Hougaard P (1984) Epidemiological studies of diabetes mellitus in Denmark: 5. Mortality and causes of death among insulin-treated diabetic patients. Diabetologia 26: 190194

13. Uemura K, Zbynek P (1988) Trends in cardiovascular disease mortality in industrialized countries since 1950 . World Health Statistics Quarterly 41: 155-178

14. Westlund K (1969) Mortality of diabetics. University Press, Norway

Received: 22 February 1990

and in revised form: 2 July 1990

Dr. G. Joner

Pediatric Department

Aker University Hospital

$\mathrm{N}-0514$ Oslo 5

Norway 